\title{
Targeted immunology for prevention and cure of VL
}

\author{
1 Infectious Diseases and Immunology, Indian Institute of Chemical Biology, Kolkata, India \\ 2 US Food and Drug Administration, Silver Spring, MD, USA \\ ${ }^{3}$ National Institute of Allergy and Infectious Diseases, Rockville, MD, USA \\ ${ }^{4}$ Universidade Federal de Ouro Preto, Ouro Preto, Brazil \\ ${ }^{*}$ Correspondence: nali@iicb.res.in \\ Edited and reviewed by: \\ Kendall A. Smith, Weill Medical College of Cornell University, USA
}

Nahid Ali ${ }^{1}$ *, Hira L. Nakhasi ${ }^{2}$, Jesus G. Valenzuela ${ }^{3}$ and Alexandre Barbosa Reis ${ }^{4}$

Keywords: visceral leishmaniasis, immunotherapy, vaccine, immuno-modulator, Th1 response

Leishmaniasis is a neglected tropical disease caused by a group of protozoan parasites of the genus Leishmania. Clinical presentation of leishmaniasis can range from cutaneous, mucocutaneous, or visceral forms depending on the parasite species. Visceral leishmaniasis (VL) caused by L. donovani and L. infantum is the severest and one of the deadliest parasitic diseases of the tropics second only to malaria (1). Nearly, 20,000-40,000 annual deaths are estimated due to this disease (2). Except for the Indian subcontinent and West Africa, VL is frequent in dogs, which serve as the major reservoir for zoonosis (3).

Transmitted by the bite of an infected sandfly, Leishmania endure in the phagolysomal compartment of macrophages by evasion and attenuation of the microbicidal functions of the host $(4,5)$. Leishmania has evolved as a successful parasite chiefly by its ability to modulate the immunological and cyto-chemical responses of the host following infection. The key strategy for successful pathogenesis is to subvert the nitric oxide burst in the host macrophage. This opportunistic parasite thus establishes a safe niche in the inactivated phagocyte and uncontrolled parasitization in liver, spleen, and bone marrow leads to symptomatic VL characterized by fever, weight loss, hepatosplenomegaly, and anemia (3).

Since the pathogenesis of the disease is based on subversion and modulation of both innate and adaptive arms of immunity, the disease is opportunistic to immuno-suppression (6). Hence, commencement of an appropriate immune response is a challenge for the control of VL infection. Indeed, therapeutic drugs like SSG and miltefosine are immuno-modulators that trigger Th1 responses essential for activation of oxidative burst in the macrophages (7, 8). However, major limitations of narrow therapeutic index and increasing incidence of resistance with currently used drugs for VL are encumbrances in effective disease management. Recent approaches like combination therapy, targeted delivery, and use of immune-adjunct are efforts to bring down the effective doses of these toxicity-associated drugs. Most promising are the prospects of various immune-targeted therapeutic approaches for treatment of VL (9). Various leishmanial antigens, cytokines, and antibodies that initiate protective Th1-biased cell-mediated immune responses used singly or as an adjunct to conventional chemotherapy are potent immuno-chemotherapeutic agents for the cure of VL $(10,11)$. Additionally, medicinal plants and their products have opened new dimensions in search of safer and cheaper anti-leishmanial immuno-modulators. These phytochemicals are not only promising as immuno-chemotherapeutic agents against VL but also have potential as immuno-adjuvants and adjuncts to chemotherapy for a number of other immuno-regulatory diseases (12).

Since both cure and resilience to Leishmania infection depend on the immunological status of the host, the antigens that can trigger healing responses can also induce prophylactic immunity. Therefore, identification of immunogens that can induce Th1 responses is the critical aspect of vaccine search against VL (13).

Although a number of defined antigens have been reported to impart protective immunity against experimental VL, recent trend of reverse vaccinology is a promising aspect for identification of key immunogens for a successful vaccine $(13,14)$. This requires rational inputs and algorithm for identification of a promising antigen from the whole proteome data analysis in silico (15). One of the key inputs is to identify the epitopes for activation of both $\mathrm{CD} 4^{+} \mathrm{Th} 1$ and $\mathrm{CD} 8^{+} \mathrm{T}$ cells. Indeed, several studies have attempted to generate epitope-based vaccines from potent antigens that selectively targets MHC I and MHC II. These multi-epitope-based synthetic vaccines were found to stimulate Th1 and $\mathrm{CD} 8^{+} \mathrm{T}$ cell responses and can be potentially used for prophylaxis against VL $(16,17)$. Since antigen presenting cells determine the activation of specific lymphocyte subsets, targeting dendritic cells that are known for activation of Th1 and $\mathrm{CD}^{+} \mathrm{T}$ cells can serve as an important vaccination strategy against VL. Indeed, various reports of antigen-pulsed DCs as vaccine against experimental VL have been promising (18).

The vector (sandfly) salivary proteins play a pivotal role in parasite pathogenesis. Indeed, the infective dose of Leishmania parasites during natural transmission is much lower as compared to saliva free infectious inoculums (19). This has been primarily attributed to the initial immune responses to salivary component triggered following sandfly bite, which enhances the infectivity of Leishmania in the host. Rationally, priming the host against a number of sandfly salivary proteins have been shown to induce altered host immunity to the parasite imparting protection against Leishmania infection $(20,21)$. Therefore, salivary proteins alone or in combination with parasite antigens can be promising vaccine components against VL (22).

However, most defined protein based vaccines are limited by their inability to generate profound long lasting immunity. This is in part due to lack of antigen persistence and multiplicity of 
antigens required to generate long lasting memory without a suitable adjuvant (23). In fact, lifelong immunity gained through natural infection is the gold standard of protection for VL. Therefore, apart from triggering appropriate immune responses, the immune correlates of long lasting protective immunity have to be determined. This can be achieved in part by the partial mimic of natural infection, which ensures antigen/parasite persistence and multi-antigenicity required for robust long lasting immunity. Although DNA vaccines can ensure antigen persistence, it is limited by multiplicity of antigens required and the potential adverse effects associated.

For this very reason, several genetically modified live parasites have been found to be the most efficient vaccination strategy (23, 24). However, despite reported success as a vaccination strategy against experimental VL, none of the genetically modified organisms have been approved for clinical trials. The primary concern is the safety issue associated with live parasites. Possibility of revert pathogenesis makes the use of live parasites speculative for human administration. However, understanding the biomarkers of safety of the live vaccines in human cell can be highly valuable in the development of a successful vaccine against VL (25).

\section{ACKNOWLEDGMENTS}

We would like to thank all support staff at the Frontiers Editorial Office for their guidance and cooperation and most importantly we are thankful to the authors for their valuable contributions to this research topic. We are also indebted to the timely and expert review by all the reviewers.

\section{REFERENCES}

1. Stauch A, Duerr HP, Picado A, Ostyn B, Sundar S, Rijal S, et al. Model-based investigations of different vector-related intervention strategies to eliminate visceral leishmaniasis on the Indian subcontinent. PLoS Negl Trop Dis (2014) 8(4):e2810. doi:10.1371/journal.pntd.0002810

2. Alvar J, Velez ID, Bern C, Herrero M, Desjeux P, Cano J, et al. Leishmaniasis worldwide and global estimates of its incidence. PLoS One (2012) 7(5):e35671. doi:10.1371/journal.pone.0035671

3. Palatnik-de-Sousa CB. Vaccines for canine leishmaniasis. Front Immunol (2012) 3:69. doi:10.3389/fimmu.2012.00069

4. Kaye P, Scott P. Leishmaniasis: complexity at the host-pathogen interface. Nat Rev Microbiol (2011) 9(8):604-15. doi:10.1038/nrmicro2608

5. Cecilio P, Perez-Cabezas B, Santarem N, Maciel J, Rodrigues V, Cordeiro da Silva A. Deception and manipulation: the arms of Leishmania, a successful parasite. Front Immunol (2014) 5:480. doi:10.3389/fimmu.2014.00480

6. Kumar R, Nylen S. Immunobiology of visceral leishmaniasis. Front Immunol (2012) 3:251. doi:10.3389/fimmu.2012.00251

7. Fan K, Borden E, Yi T. Interferon-gamma is induced in human peripheral blood immune cells in vitro by sodium stibogluconate/interleukin-2 and mediates its antitumor activity in vivo. J Interferon Cytokine Res (2009) 29(8):451-60. doi:10.1089/jir.2008.0061

8. Wadhone P, Maiti M, Agarwal R, Kamat V, Martin S, Saha B. Miltefosine promotes IFN-gamma-dominated anti-leishmanial immune response. J Immunol (2009) 182(11):7146-54. doi:10.4049/jimmunol.0803859

9. Singh OP, Sundar S. Immunotherapy and targeted therapies in treatment of visceral leishmaniasis: current status and future prospects. Front Immunol (2014) 5:296. doi:10.3389/fimmu.2014.00296

10. Nico D, Gomes DC, Palatnik-de-Sousa I, Morrot A, Palatnik M, Palatnik-deSousa CB. Leishmania donovani nucleoside hydrolase terminal domains in crossprotective immunotherapy against Leishmania amazonensis murine infection. Front Immunol (2014) 5:273. doi:10.3389/fimmu.2014.00273

11. Roatt BM, Aguiar-Soares RD, Coura-Vital W, Ker HG, Moreira N, Vitoriano-Souza J, et al. Immunotherapy and immunochemotherapy in visceral
Leishmaniasis: promising treatments for this neglected disease. Front Immunol (2014) 5:272. doi:10.3389/fimmu.2014.00272

12. Chouhan G, Islamuddin M, Sahal D, Afrin F. Exploring the role of medicinal plant-based immunomodulators for effective therapy of leishmaniasis. Front Immunol (2014) 5:193. doi:10.3389/fimmu.2014.00193

13. Joshi S, Rawat K, Yadav NK, Kumar V, Siddiqi MI, Dube A. Visceral Leishmaniasis: advancements in vaccine development via classical and molecular approaches. Front Immunol (2014) 5:380. doi:10.3389/fimmu.2014.00380

14. Nico D, Gomes DC, Alves-Silva MV, Freitas EO, Morrot A, Bahia D, et al. Crossprotective immunity to Leishmania amazonensis is mediated by CD4+ and CD8+ epitopes of Leishmania donovani nucleoside hydrolase terminal domains. Front Immunol (2014) 5:189. doi:10.3389/fimmu.2014.00189

15. Aebischer T. Leishmania spp. proteome data sets: a comprehensive resource for vaccine development to target visceral leishmaniasis. Front Immunol (2014) 5:260. doi:10.3389/fimmu.2014.00260

16. Das S, Freier A, Boussoffara T, Oswald D, Losch FO, Selka M, et al. Modular multiantigen T cell epitope-enriched DNA vaccine against human leishmaniasis. $\mathrm{Sci}$ Transl Med (2014) 6(234):234ra56. doi:10.1126/scitranslmed.3008222

17. Agallou M, Athanasiou E, Koutsoni O, Dotsika E, Karagouni E. Experimental validation of multi-epitope peptides including promising MHC class I- and IIrestricted epitopes of four known Leishmania infantum proteins. Front Immunol (2014) 5:268. doi:10.3389/fimmu.2014.00268

18. Majumder S, Bhattacharjee A, Paul Chowdhury B, Bhattacharyya Majumdar S, Majumdar S. Antigen-pulsed CpG-ODN-activated dendritic cells induce hostprotective immune response by regulating the $t$ regulatory cell functioning in Leishmania donovani-infected mice: critical role of CXCL10. Front Immunol (2014) 5:261. doi:10.3389/fimmu.2014.00261

19. Titus RG, Ribeiro JM. Salivary gland lysates from the sand fly Lutzomyia longipalpis enhance Leishmania infectivity. Science (1988) 239(4845):1306-8. doi:10.1126/science. 3344436

20. Gomes R, Oliveira F, Teixeira C, Meneses C, Gilmore DC, Elnaiem DE, et al. Immunity to sand fly salivary protein LJM11 modulates host response to vector-transmitted Leishmania conferring ulcer-free protection. J Invest Dermatol (2012) 132(12):2735-43. doi:10.1038/jid.2012.205

21. Gomes R, Teixeira C, Teixeira MJ, Oliveira F, Menezes MJ, Silva C, et al. Immunity to a salivary protein of a sand fly vector protects against the fatal outcome of visceral leishmaniasis in a hamster model. Proc Natl Acad Sci U S A (2008) 105(22):7845-50. doi:10.1073/pnas.0712153105

22. Kamhawi S, Aslan H, Valenzuela JG. Vector saliva in vaccines for visceral leishmaniasis: a brief encounter of high consequence? Front Public Health (2014) 2:99. doi:10.3389/fpubh.2014.00099

23. Saljoughian N, Taheri T, Rafati S. Live vaccination tactics: possible approaches for controlling visceral leishmaniasis. Front Immunol (2014) 5:134. doi:10.3389/ fimmu.2014.00134

24. Chhajer R, Ali N. Genetically modified organisms and visceral leishmaniasis. Front Immunol (2014) 5:213. doi:10.3389/fimmu.2014.00213

25. Gannavaram S, Dey R, Avishek K, Selvapandiyan A, Salotra P, Nakhasi HL. Biomarkers of safety and immune protection for genetically modified live attenuated Leishmania vaccines against visceral leishmaniasis - discovery and implications. Front Immunol (2014) 5:241. doi:10.3389/fimmu.2014.00241

Conflict of Interest Statement: The authors declare that the research was conducted in the absence of any commercial or financial relationships that could be construed as a potential conflict of interest.

Received: 21 November 2014; accepted: 08 December 2014; published online: 19 December 2014.

Citation: Ali N, Nakhasi HL, Valenzuela JG and Reis AB (2014) Targeted immunology for prevention and cure of VL. Front. Immunol. 5:660. doi: 10.3389/fimmu.2014.00660 This article was submitted to Immunotherapies and Vaccines, a section of the journal Frontiers in Immunology.

Copyright (c) 2014 Ali, Nakhasi, Valenzuela and Reis. This is an open-access article distributed under the terms of the Creative Commons Attribution License (CC BY). The use, distribution or reproduction in other forums is permitted, provided the original author(s) or licensor are credited and that the original publication in this journal is cited, in accordance with accepted academic practice. No use, distribution or reproduction is permitted which does not comply with these terms. 